\title{
Acompañamiento pedagógico y desempeño docente en educación primaria
}

\section{Pedagogical accompaniment and teaching performance in primary education}

\author{
VEGA, Pedro G. ${ }^{1}$ \\ VASQUEZ, Cristina Y. ${ }^{2}$

\section{Resumen} \\ El objetivo de estudio fue establecer la relación entre el acompañamiento pedagógico y desempeño \\ docente en educación primaria; se empleó la investigación bibliográfica, valiéndose de métodos \\ como:análisis-síntesis, generalización y abstracción, así como el interpretativo y contrastación empírica. \\ Los resultados evidencian deficiencias en relación a las variables, siendo necesario apoyarse en la \\ experiencia del directivo y del docente para alcanzar un nivel satisfactorio. En conclusión, existe la \\ presencia de relación estrecha entre el acompañamiento pedagógico y desempeño docente. \\ Palabras clave: acompañamiento pedagógico, desempeño docente, enseñanza aprendizaje
}

\begin{abstract}
The objective of the study was to establish the relationship between pedagogical accompaniment and teaching performance in primary education; Bibliographic research was used, using methods such as: analysis-synthesis, generalization and abstraction, as well as interpretive and empirical contrasting; The results show deficiencies in relation to the variables, being necessary to rely on the experience of the manager and the teacher to reach a satisfactory level. In conclusion, there is a close relationship between pedagogical support and teaching performance.

key words: pedagogical accompaniment, teaching performance, teaching-learning
\end{abstract}

\section{Introducción}

En el presente estudio se abordan variables como el acompañamiento pedagógico y el desempeño docente, pues se considera que la educación debe responder a exigencias y demandas de la sociedad en la actualidad, para garantizar que se imparta una educación de calidad, que conlleve a formar personas competentes que se adapten a los cambios, y puedan resolver los retos que se presentan en su entorno social. Bajo esa concepción se observa que a nivel mundial se han desarrollado estudios sobre esta temática, sin embargo, existe dificultad por parte de los directivos en las instituciones para realizar el acompañamiento a sus docentes, con miras a mejorar su desempeño. Al respecto, Rivas (2017) indica que los directivos institucionales, como líderes pedagógicos, se encargan de acompañar a los docentes para desarrollar sus competencias profesionales, lo que

\footnotetext{
${ }^{1}$ Especialista en Educación. Ancash. Universidad César Vallejo. Perú. pgvegaa@ucvvirtual.edu.pe

2 Especialista en Educación. Ancash. Universidad César Vallejo. Perú. cyvasquezc@ucvvirtual.edu.pe
} 
permite el enriquecimiento del ejercicio docente. Por otro lado, Day, et al. (2010), afirma que el desempeño experto de los instructores se encuentra centrado en la mejora continua de los enfoques de aprendizaje, que se generan a través de actividades oportunas y consistentes desarrolladas por los estudiantes. En este sentido, naciones avanzadas como Japón y Estados Unidos, entre otros, han entendido que el acompañamiento al educador ayuda a cosechar mejores resultados en lo académico; además, las prácticas relacionadas con la asistencia pedagógica a los instructores son vitales para un proceso de desarrollo docente eficaz y continuo (Johnston, Kaufman y Thompson, 2016).

En Perú, el desempeño docente tiene un nivel bajo, según resultados de una evaluación realizada por el Ministerio de Educación sobre el desempeño docente para el ascenso de escala en el año 2018, el 83\% de los profesores obtuvieron resultados desaprobatorios (Cuenca, 2020). Por tanto, existe la preocupación de cómo promover estrategias para establecer el acompañamiento pedagógico a los docentes como apoyo en su labor didáctica, de manera que los fortalezca en los aspectos de planificación curricular, manejo de estrategias, empleo de materiales tecnológicos y evaluación formativa (Contreras, 2016). Se entiende al acompañamiento pedagógico como elemento vital para gestionar ambientes de aprendizaje colaborativo en cada institución, que permitan la deconstrucción y reflexión sobre la práctica pedagógica de los docentes para promover la mejora de la calidad de los aprendizajes en los estudiantes (Vezub y Alliaud, 2012).

En la jurisdicción de la Ugel Santa, en la provincia del Santa, Región Ancash, los directivos y los docentes que trabajan en instituciones públicas de su influencia, buscan regularmente mejorar su práctica pedagógica para beneficiar a sus educandos; sin embargo, los resultados y las decisiones tomadas como consecuencia del seguimiento no se contemplan o no favorecen las tareas instructivas en Educación Primaria, lo cual hace suponer la relación entre ambas variables. Marcelo y Vaillant (2009) refieren que el desempeño profesional del docente es importante en el complejo proceso de enseñar y aprender, por lo tanto, los sistemas educativos mejorarán cuando se cuente con docentes con alto nivel de preparación para las actividades de enseñar, y cuando éstos tengan la convicción de que sus estudiantes pueden efectivamente aprender. Ante lo mencionado, se formula como problema de investigación la siguiente interrogante ¿Cuál es la relación entre el acompañamiento pedagógico y el desempeño docente en Educación Primaria? Frente a la cual, se tiene como propósito efectuar una revisión descriptiva de la literatura que nos conceda identificar la relación entre las variables de estudio acompañamiento pedagógico y el desempeño docente.

Considerando las tareas establecidas para el desarrollo de la investigación una de las primeras acciones fue la identificación de antecedentes en la misma línea temática, rescatándose los aportes de Leyva y Vásquez (2019), Díaz (2018), Agreda y Perez (2020) que refieren que el acompañamiento pedagógico es brindado por el directivo en apoyo al docente durante el proceso de enseñanza, esto se gestiona a través de una formación continua y las variables tienen una relación directa, percibida por los docentes en las instituciones que fueron evaluados; además de encontrarse en un nivel destacado.

Por otro lado, dentro de la realidad peruana, Martínez (2016), Quijano (2020) y Villalobos (2019) plantean que existe una relación directa y significativa entre las variables de estudio y mencionan que es necesario la participación activa de los involucrados en el acompañamiento y la aplicación de estrategias que influyen directamente en el fortalecimiento del desempeño docente durante el proceso educativo, y así proporcionar un panorama actual de lo que se viene haciendo o deja de hacer en la labor pedagógica a través de la reflexión.

En complemento de la acción realizada y para dar fundamento teórico a las variables estudiadas, las definiciones de la literatura, se ubica a Montanares y Junod (2018) quienes refieren que, acompañamiento pedagógico se da a través de un intercambio recíproco de experiencias entre el maestro que acompaña a otro profesor con el propósito de guiar y fortalecer al acompañado, empleando el enfoque crítico reflexivo a través de una serie de recursos estratégicos orientados a mejorar la labor del maestro, esta tarea lo realiza observando y valorando la 
información recogida sobre el trabajo del profesor, compartiendo una reflexión crítica, con el propósito de mejorar las competencias y capacidades pedagógicas del maestro acompañado.

Asimismo, el Ministerio de Educación (2014) precisa que es una estrategia para la preparación continua y en servicio docente, la cual tiene como finalidad mejorar el trabajo pedagógico del maestro con la participación de especialistas para enfrentar los retos planteados en el Proyecto Educativo Nacional (PEN). Para este fin, el acompañante incorpora una variedad de acciones con distintos aportes teóricos a través de un enfoque crítico reflexivo. Además, Bryk y Schneider (2002) lo definen como un conjunto de fases sistemáticos y permanentes, dado por el acompañante, siendo su propósito la interacción con el director y el docente, así realizar una reflexión crítica sobre el trabajo que desempeña con sus alumnos, que permita tomar decisiones oportunas e implementar cambios en su trabajo.

Por otra parte, la observación que realiza el acompañante sobre el trabajo que realiza el docente de aula, es con el propósito de registrar, analizar e interpretar la información recogida, que ayude al docente a realizar la deconstrucción de su práctica, haciendo uso de preguntas reflexivas. En tal sentido, ambos construyen el saber pedagógico, a través de la retroalimentación y el reajuste de la práctica pedagógica. Sobre lo mencionado, Smith y Smith (2015) explican que se acompaña para ofrecer asesoría a través del desarrollo de una asistencia técnica, donde un docente o equipo especializado visita al profesor en su aula, para ofrecer asesoramiento continuo en temas importantes a su práctica; es una tarea pedagógica que se orienta a fortalecer la labor del docente a través de una asistencia técnica que permite un intercambio de experiencias entre ambos participantes, sin distinción de saber quién es más que el otro, buscando una interacción verdadera, compartiendo experiencias en un ambiente de confianza relacionado al quehacer educativo.

También, Hallinger y Wang (2015) refieren que la supervisión es una función que a diario realiza el director, con el propósito de aconsejar al profesor a través de asistencias técnicas, para fortalecer sus capacidades y competencias que permitan promover la sensibilidad y las relaciones positivas entre las personas. De igual forma, Arlestig y Tornsen (2014) sostienen que el acompañamiento compone un buen momento para impulsar espacios de aprendizaje colectivo al interior de las escuelas que impulsen la revisión y reflexión de la práctica pedagógica de los docentes con la finalidad de mejorar la calidad de los aprendizajes en los estudiantes.

Además, Day, Sammons, Stobart, Kington y Gu (2009) explican que el acompañamiento es una construcción teórica que se desarrolla entre el docente que realiza el acompañamiento y el que es acompañado, lo cual permite compartir experiencias que dan como resultado mejoras en la práctica pedagógica del docente acompañado y la comprensión de sus deberes laborales. Es una fase flexible y direccionada en un contexto global, que cobra sentido al transformarse en nuevos modelos de la práctica didáctica de los profesores, que son acompañados. Por consiguiente, Montero (2011) menciona sobre el acompañamiento pedagógico que se asume como la estrategia que tiene que ver con la preparación de los profesores a quienes el acompañante brinda asesoría personalizada al maestro acompañado, con respecto a la práctica pedagógica, iniciando desde sus necesidades formativas del acompañado, a través del diálogo reflexivo y el compromiso del docente.

Con respecto al desempeño docente, Faria, Reis y Peralta (2016) explican que es fundamental para el desarrollo del proceso educativo de los estudiantes dentro de las instancias educacionales. En ese sentido, los maestros implementan la planificación, ejecución y evaluación de un conjunto de actividades que serán desarrollados por los aprendices con apoyo de la mediación del docente. Además, el maestro debe contar con un alto nivel de preparación en habilidades y conocimientos, gestionando su formación continua para que cubra las exigencias de la sociedad de impartir una educación de calidad.

Por otra parte, Martínez y Lavín (2017), Sikes, Measor y Woods (2002) explican que el desempeño del docente constituye una serie de actividades que debe cumplir, referido a su práctica pedagógica, cuyos conocimientos 
los ha adquirido durante su preparación profesional, donde sus capacidades y competencias le permiten tener dominio de conocimientos teóricos-prácticos acerca de los aprendizajes y la conducta humana, cuyo fin es promover las relaciones positivas para desarrollar un conjunto de estrategias de enseñanza que aseguren un aprendizaje de calidad. Asimismo, Montenegro (2007) dice que es un factor primordial para la calidad educativa y por ende, la ejecución de actividades previstas en la preparación profesional de personas competentes que puedan desenvolverse con éxito en las tareas pedagógicas que se desarrollan en los colegios.

\section{Metodología}

El presente estudio se realizó a través de una revisión de literatura que permitió el acopio de información referida al acompañamiento pedagógico y sobre el desempeño docente, con la finalidad de dar respuesta al objetivo planteado. Para ello, se consideró el tipo de investigación bibliográfica. Por último, se realizó la revisión bibliográfica teniendo en cuenta el título, autores, año, país, metodología, muestra, resultados y conclusiones, lo cual ayudó a realizar el análisis de los resultados.

Asimismo, se utilizó como principal método el análisis-síntesis de las evidencias encontradas en documentos científicos, para luego llegar a generalizaciones que permitan conocer la relación vigente entre las variables asumidas como son el caso del acompañamiento pedagógico y el desempeño docente, a partir del método de generalización y abstracción, frente a los cuales se habían formulado las conclusiones que han contribuido al paso de cierre, dentro de la redacción del informe pensando en las tácticas instaladas en el objetivo del estudio.

El estudio consideró la búsqueda y selección de información bibliográfica de 80 documentos en fuentes de información escrita y web, artículos científicos, Tesis de doctorado, así como diversos libros, provenientes de revistas electrónicas como Scielo, Dialnet, Latindex y Google académico, los cuales han permitido una adecuada selección de documentos pertinentes relacionados al objeto de estudio. Se priorizó 30 literaturas bibliográficas de los años últimos, tanto en idioma español como inglés, considerando campos de reacción nacionales, latinoamericanos y europeos.

De igual modo, el artículo en su redacción ubica el método interpretativo y de contrastación empírica, que en caso del primero, orientado a la descripción de los fundamentos teóricos recopilados y analizados, que posteriormente fueron contrastados y presentados como parte de las conclusiones teóricas que se encuentran al final del presente estudio (Creswell, 2003). En el caso del análisis de la información selecta, a partir de la contrastación con el marco referencial y la experiencia empírica, se ha conducido a la formulación de las conclusiones teóricas de acuerdo con al objetivo trazado para la presente investigación.

\section{Resultados y discusión}

A continuación, se presentan los resultados de corte académico relacionados con el objetivo de identificar la relación entre las variables acompañamiento pedagógico y el desempeño docente. Según la literatura analizada existe una relación directa y significativa entre las variables de estudio y que el acompañamiento pedagógico es destacado en las instituciones de educación primaria. Asimismo, Agreda y Pérez (2020) refiere que el acompañamiento pedagógico se relaciona directamente con el desempeño docente, observando en los docentes una reflexión positiva de su práctica pedagógica con el propósito de gestionar las mejoras que contribuirán al logro de los aprendizajes en los estudiantes.

Los resultados dentro de la línea teórica, han ubicado que el soporte básico para planificar una gestión educativa de calidad parte del reconocimiento de los problemas vigentes dentro de la institución; en esa línea, de acuerdo con Díaz (2018) el modelo de gestion debe permitir representar el seguimiento de la gestión directiva, basado en los paradigmas de la elaboración de planes contemporáneos, así como el control instruccional realizado por 
los directivos, teniendo como referente el nivel de desempeño óptimo en el docente, tal como también lo señalan Leyva y Vasquez, 2019.

El estudio bibliográfico realizado también nos presenta información valiosa para un futuro inmediato, permitiendo mejorar los procesos educativos que fortalezcan el proveer a la comunidad académica y contar con un modelo de control prospectivo con propuestas de intercambio ante situaciones exigentes recientes; al respecto Quijano (2020), sostiene que la naturaleza de estos estudios deben contribuir a la construcción de planes estratégicos que propicien una adecuada capacidad de gestión educativa, para ofertar calidad educativa en la vida de los estudiantes. Asimismo, se resalta como resultado del estudio la importancia del acompañamiento pedagógico dentro de la Gestión educativa más cercana al éxito de los retos primarios de la organización, optando por una selección educativa precisa, coincidiendo con los objetivos de la política educativa de actualidad (Aguirre y Porta, 2019).

En los resultados también se considera que el estudio ha contribuido al enriquecimiento del saber hacer del investigador en temas instruccionales, impulsando, a través de la investigación, un aporte al desarrollo de la acción docente y por tanto, ofrecer una gran educación (Ortega, 2019), situación que favorecerá la elaboración de un plan estratégico que permite al formador del grupo académico potenciar sus labores pedagógicas considerando los deseos y rasgos de los estudiantes, con la guía de un trabajo planificado que conduzca a adquirir mejores resultados dentro de los procesos de gestión institucional y pedagógica, beneficios que recaen de inmediato en los académicos que recibirán una educación maravillosa y placentera, y no directamente a la sociedad, donde madre y padre y la comunidad en general pueden tener la certeza de que sus hijos han adquirido los requisitos de primera clase que exigen (Anijovich y Capelletti, 2018).

El presente estudio partió de la necesidad de establecer los resultados del acompañamiento pedagógico desarrollado actualmente a través de gerentes en instituciones instruccionales de Educación Básica Regular, relacionándolos con el desempeño de los docentes, cuyo análisis que parte de los resultados lleva a señalar que el personal directivo de la institución se encuentra en proceso de actualización sobre la aplicación de la estrategia de acompañamiento pedagógico en entornos virtuales dada la coyuntura actual de la educación (Agreda y Pérez, 2020). En este caso la experiencia de los directivos relacionada a la estrategia, sumada a las capacidades de los docentes en las instituciones hacen que la gestión en algunas instituciones alcance un gran nivel; sin embargo, se toma en consideración que se debe complementar con movimientos que incluyan intercambio verbal de resultados y toma de decisiones, entre otras técnicas que lleven a los docentes junto con los directores a contar con compromisos de mejoramiento para obtener la calidad institucional educativa (Aguirre y Porta, 2019).

Así también, se indica que estos resultados evidencian relación con lo indicado por Martínez (2016) quien refiere que reconstruir la práctica de gestión permite a los directivos determinar y aplicar estrategias de acompañamiento pedagógico que se orienten a mejorar los desempeños de los docentes; sobre lo afirmado Quijano (2020) menciona que para que en las instituciones exista calidad educativa se debe contar con un adecuado planeamiento estratégico, el cual, cuando se realiza de forma adecuada, según el Ministerio de Educación (2014) favorece la recopilación de información y su posterior análisis que conlleve a contar con evidencias que permitan mejorar los procesos educativos y pedagógicos que se desarrollan en las aulas del nivel Primaria.

Referente al desempeño docente cabe resaltar que su labor ha perdido reconocimiento por parte de la sociedad, en escenarios actuales se ve expuesto a críticas por parte de padres de familia (Martínez, Esparza y Gómez, 2020). En los últimos años el Ministerio de Educación a través de los resultados de sus evaluaciones ha permitido a los instructores generar compromisos de intercambio, de mejorar sus actividades y labores pedagógicas como parte de la gestión de la institución, donde la fortaleza es el trabajo en equipo, círculos de interaprendizaje, lo que sugiere sus logros dentro del desempeño general de los estudiantes (Villalobos, 2019). 
De igual modo, Martínez y Lavín (2017) señalan que la formación de primer nivel es obligación de los directores y profesores; asimismo, refiere que la elaboración de planes estratégicos dentro de los establecimientos, el buen uso de los activos institucionales y los logros alcanzados se ven reflejados dentro de la mejora continua de las Instituciones.

\section{Conclusiones}

Los docentes de educación primaria son conscientes de sus dificultades en cuanto a procesos referidos a la planificación, implementación, ejecución y evaluación enmarcados en los cambios vertiginosos implementados por el Ministerio de Educación, asimismo, los resultados alcanzados en las evaluaciones en los últimos años denotan una necesidad de mejora, la cual debe tener su punto de partida en el adecuado acompañamiento pedagógico que no está siendo la más acertada en tiempos actuales de una educación remota.

Respecto a la labor pedagógica desarrollada por los docentes, es evidente que la necesidad actual ha hecho que resalten el compromiso y formación continua; la sociedad respalda la actitud que muestran los docentes de Primaria para favorecer aprendizajes en sus estudiantes a pesar de las adversidades que encuentran, siendo necesario por tanto informar oportunamente al docente de las debilidades encontradas durante el acompañamiento, con la intención de propiciar un incremento en su sentido de identidad profesional, que conlleve a una buena práctica pedagógica a través de planes de trabajo, proyectos y otros, en concordancia con las aspiraciones de los educandos, en respuesta a las necesidades del contexto.

Finalmente, por los fundamentos teóricos analizados y la experticia del investigador, se puede afirmar la existencia de una relación estrecha entre el acompañamiento pedagógico y el desempeño de los docentes, por lo cual se asume que el proceso de seguimiento de la labor docente, llamado acompañamiento pedagógico en los últimos años, representa un aporte pedagógico que los instructores expanden bajo la observación del director. Este proceso permite tener directivos comprometidos y competentes con la formación de los estudiantes, que ya no temen más al elemento administrativo, pero también son fortaleza para el elemento pedagógico; asimismo, es fundamental que los docentes tengan la predisposición que contribuya con la identificación de sus deficiencias comprobadas, que pida sugerencias por mejorar, asumiendo por tanto que la calidad del servicio educativo depende del compromiso de ambos actores educativos.

\section{Referencias bibliográficas}

Agreda A. y Pérez M. (2020). Relación entre acompañamiento pedagógico y práctica reflexiva. Espacios en Blanco. Revista de Educación, N 30, vol. 2, jul./dic. 2020, pp. 219-232. Universidad Nacional del Centro de la Provincia de Buenos Aires, Tandil, Argentina. http://www.scielo.org.ar/pdf/eb/v30n2/2313-9927-eb-3002-1.pdf

Aguirre J. y Porta L. (2019). La formación docente con rostro humano. Tensiones y desafíos polifónicos desde una perspectiva biográfico-narrativa. Revista Espacios en Blanco, 1(29), Enero-Junio, 161 - 182. https://www.redalyc.org/jatsRepo/3845/384556936010/384556936010.pdf

Anijovich R. y Capelletti G. (2018). La práctica reflexiva en los docentes en servicio. Posibilidades y limitaciones. Revista Espacios en Blanco, (28), 74-92. http://www.redalyc.org/jatsRepo/3845/384555587005/384555587005.pdf

Arlestig H. \& Tornsen M. (2014). Classroom observations and supervision-essential dimensions of pedagogical leadership. International Journal of Educational Management, 28(7), 856-868. https://www.emerald.com/insight/content/doi/10.1108/IJEM-01-2014-0001/full/html 
Bryk A.S. \& Schneider B.L. (2002). Trust in schools: a core source for improvement. Russell Sage Foundation Publications.

Contreras T. (2016). Liderazgo pedagógico, liderazgo docente y su papel en la mejora de la escuela: una aproximación teórica. Propósitos y Representaciones, 4(2): 231-284.

https://revistas.usil.edu.pe/index.php/pyr/article/view/123

Creswell J.W. (2003). Research: Qualitative, Quantitative and Mixed methods approaches. Segunda edición. Sage.

Cuenca R. (2020). La evaluación docente en el Perú (1ra ed.). Perú: Instituto de estudios peruanos. Obtenido de http://repositorio.iep.org.pe/handle/IEP/9

Day C., Sammons P., Leithwood K., Hopkins D., Harris A., Gu Q., \& Brown E. (2010). Ten strong claims about successful school leadership. Nottingham: NCSL. https://www.gov.uk/government/publications/10-strongclaims-about-successful-school-leadership

Day C., Sammons P., Stobart G., Kington A. \& Gu Q. (2009). Teachers matter: variations in work, lives and effectiveness. Open University Press.

Díaz M. (2018). Modelo de gestión del acompañamiento pedagógico para maestro de primaria. Transformación, 14(1), 44-53. http://scielo.sld.cu/scielo.php?script=sci_arttext\&pid=S207729552018000100005\&lng=es\&tlng=es.

Faria A., Reis P. y Peralta H. (2016). La formación de profesores: ¿formación continua o formación postgraduada? Perspectivas de profesores y de líderes de las escuelas. Revista Electrónica Interuniversitaria de Formación del Profesorado, 19(2), 289-296. https://revistas.um.es/reifop/article/view/254891

Hallinger P. \& Wang W. C. (2015). Assessing instructional leadership with the principal instructional management rating scale. Dordrecht: Springer.

Johnston W.R., Kaufman J.H. \& Thompson L. E. (2016). Support for instructional leadership. http://www.wallacefoundation.org/knowledgecenter/Documents/Support-for-InstructionalLeadership.pdf

Leyva M. y Vasquez C. (2019). Liderazgo pedagógico: de la supervisión al acompañamiento docente. Calidad en la Educación(51), Dic 2019, № 51 Paginas 225 - 251. doi:https://doi.org/10.31619/caledu.n51.635 https://scielo.conicyt.cl/pdf/caledu/n51/0718-4565-caledu-51-225.pdf

Marcelo C. \& Vaillant D. (2009). Desarrollo profesional docente. ¿Cómo se aprende a enseñar? Madrid: Nancea S. A. de ediciones.

Martínez G. (2016). El desempeño docente y la calidad Educativa. Ra Ximhai, 12(6), 123-134. https://www.redalyc.org/artículo.oa?id=461/46148194007

Martínez G.; Esparza A.; Gómez, R. (2020). El desempeño docente desde la perspectiva de la práctica profesional. RIDE. Revista Iberoamericana para la Investigación y el Desarrollo Educativo Dic 2020, Volumen 11 № 21 elocation e013 http://www.scielo.org.mx/pdf/ride/v11n21/2007-7467-ride-11-21e013.pdf 
Martínez S. y Lavín J. (2017). Aproximación al concepto de desempeño docente, una revisión conceptual sobre su delimitación. En Congreso nacional de investigación educativa - COMIE. Congreso llevado a cabo en San Luis Potosí. http://www.comie.org.mx/congreso/memoriaelectronica/v14/doc/2657.pdf

Ministerio de Educación (2014). Protocolo de acompañamiento pedagógico (1ra ed.). MACOLE S.R.L.

Montanares E. y. Junod P. (2018). Creencias y prácticas de enseñanza de profesores universitarios en Chile. Revista electrónica de investigación educativa, 20(1): 93-103. http://www.scielo.org.mx/scielo.php?script=sci_arttext\&pid=S1607-40412018000100093

Montenegro I. (2007). Evaluación de desempeño docente: Fundamentos, modelos e instrumentos. Cooperativa editorial magisterio.

Montero C. (2011). Estudio sobre acompañamiento pedagógico. Experiencias, orientaciones y temas pendientes. Consejo Nacional de Educación.

Ortega C. (2019). La reflexión como proceso en las prácticas docentes e investigativas. Diálogos sobre educación. Temas actuales en investigación educativa, 10(18), Enero-Junio, 01-05. http://www.redalyc.org/jatsRepo/5534/553458251012/553458251012.pdf

Quijano M. (2020). Acompañamiento pedagógico directivo y el desempeño docente.Institución educativa Monseñor Fidel Olivas Escudero. Ancash 2019. Tesis Doctoral, Universidad Cesar Vallejo, Ancash, Perú. https://hdl.handle.net/20.500.12692/44316

Rivas A. (2017). Cambio e innovación educativa. Santillana.

Sikes, P., Measor, L. and Woods, P. (2002). Teacher Career: Crisis and Continuities. Falmer Press. https://link.springer.com/chapter/10.1007/978-90-481-2358-2_2

Smith, J.R. \& Smith, R.L. (2015). Evaluating Instructional Leadership. Recognized Practices for Success. Corwin Press.

Vezub L., \& Alliaud A. (2012). El acompañamiento pedagógico como estrategia de apoyo y desarrollo profesional de los docentes nóveles. Uruguay: OEI. Obtenido de http://www.ub.edu/obipd/wpcontent/uploads/2020/03/Noveles.pdf

Villalobos E. (2019). Acompañamiento pedagógico en el desempeño docente de instituciones educativas de jornada escolar completa, provincia de Rioja 2018. Tesis de Doctorado, Universidad César Vallejo, Perú. http://repositorio.ucv.edu.pe/handle/UCV/37311

\section{(cc) BY-NC}

Esta obra está bajo una Licencia Creative Commons

Atribución-NoComercial 4.0 Internacional 